\title{
Ensuring the aerodynamic stability of the long- span bridges through studies in the wind tunnel
}

\author{
Evgenii Khrapunov ${ }^{1, *}$, and Sergei Solovev ${ }^{2}$ \\ ${ }^{1}$ Peter the Great St. Petersburg Polytechnic University, Polytechnicheskaya st. 29, St. Petersburg, \\ 195251, Russian Federation \\ ${ }^{2}$ Krylov State Research Centre, laboratory of Aerodynamics, Moskovskoe shosse 44, St. Petersburg, \\ 196158, Russian Federation
}

\begin{abstract}
The main ideas of the aerodynamic studies of large bridges are presented in present paper. Main types of aero-elastic instability for bridges with spans over 100 meters are considered. A two-step modeling approach is presented. At the first stage, the aerodynamic characteristics of the span fragment are considered, at the second stage - the characteristics of the whole bridge. Methods for investigation of bridge oscillations in a special-purpose experimental facility - the Landscape Wind Tunnel - are described. Examples of tests with elastic similar models of bridges are given, and measurements to mitigate dangerous oscillations early in the bridge design process are described.
\end{abstract}

\section{Introduction}

In the design of long-span bridges it is particularly important to investigate aerodynamic stability of these structures under wind loads. It is well known that bridges with spans over 100 meters are subject to aero-elastic oscillations of large amplitudes that may well result in structural failures [1].

The Volgograd Bridge galloping in Russia near the city of Volgograd is a vivid example of prohibitively intensive oscillations caused by vortex resonance, while the Tacoma Narrows Bridge near the city of Tacoma disaster in USA is an evidence of catastrophic structural failures caused by such phenomena [2].

Today, practically all long-span bridges have to pass aerodynamic tests intended to find and remedy any negative aerodynamic effects; for this reason no fresh cases of bridge failures under wind loads have been reported [3-6]. Such experimental verifications of unique bridges are performed in special wind tunnels.

Main factors causing aero-elastic oscillations of bridges and their structural components under wind loads are:

- wind gusts;

- periodic aerodynamic forces induced by Karman vortex street;

- negative aerodynamic damping resulting in self-sustained oscillations;

- aerodynamic cross-coupling of bending and torsional strains;

\footnotetext{
* Corresponding author: hrapunov.evgenii@gmail.com
} 
- periodic variation of system's parameters.

Aero-elastic oscillations are characterized by two principal parameters: critical velocity causing oscillations and maximum amplitude of such oscillations. Early design analysis of whether the structure in question is prone to aerodynamic instability or not can be based on the following approaches and features.

Let us consider in detail the main types of aerodynamic instability of large-span bridges.

Vortex excitation - oscillations arising when the natural frequency coincides with the Karman vortex shedding frequency [7]. As a rule, the critical wind velocity that excites the first natural frequency is in the range of 10 to $20 \mathrm{~m} / \mathrm{s}$, being a common recurring velocity over the service lifetime. Cyclic vortex-generated load and associated number of loading cycles can be quite important for structural analysis.

Bending-torsion flutter, i.e. coupled bending-torsion oscillations increasing with time, which are caused by mismatch between the aerodynamic center (point of aerodynamic force application) and the center of gravity [8].

In some cases structures may be disposed to aero-elastic instability of the stall flutter type, i.e. torsional oscillations due to periodic vortex shedding.

Stall flutter is characterized by [9]:

- limited amplitudes depending on the flow velocity, natural torsional frequency and torsional damping properties of structures;

- stall flutter frequency is close to one of the natural torsional frequencies of the structure;

- stall flutter critical velocity is generally lower than the critical velocity of bendingtorsional flutter.

Divergence, i.e static aero-elastic instability due to torsional aerodynamic moment [10].

Galloping, i.e self-sustained across-wind oscillations due to negative aerodynamic damping. This type of aero-elastic oscillations is characteristic of bodies with non-round cross sections as well as ice-laden stay cables. In case of galloping the oscillation amplitudes increase with the critical velocity.

Buffeting, i.e. aero-elastic instability of a structure placed in turbulent flow or in wake flow of another structure [11]. Buffeting is observed when bridges are placed close to each other.

\section{Methods of aerodynamic investigations for long-span bridges}

There is a wide variety of approaches used to generate simplified mathematical models of body oscillations in wind. However, such models are not quite fit for practical applications, being based on too many simplifications and assumptions to address the real bridge structures.

Here it is pertinent to specially mention the numerical simulation methods of Computational Fluid Dynamics (CFD), which have been rapidly developing over the last two decades. However, correct simulation of bridge oscillations in wind by CFD tools would require huge computational resources. The point is that this coupled aerodynamic and strength problem should be solved as a non-stationary problem with a very small time step. This statement of the problem calls for time-consuming computations and large errors. Therefore, CFD analysis is not used to address practical tasks either because of accuracy issues.

In this context, the physical modeling in wind tunnel facilities is found to be the key method to identify potential oscillatory behavior of bridges under wind effects. Unique long-span bridge structures are commonly investigated in two steps. At first a section model of the central span structure is made to reproduce the geometry as well as mass and inertial properties of the real structure $[12,13]$. This section model is mounted on a special- 
purpose dynamic rig in the wind-tunnel test section to examine the aerodynamic stability of the span structure. The Krylov State Research Centre is testing bridge section models in its wind tunnel of open test-section type (fig. 1a).

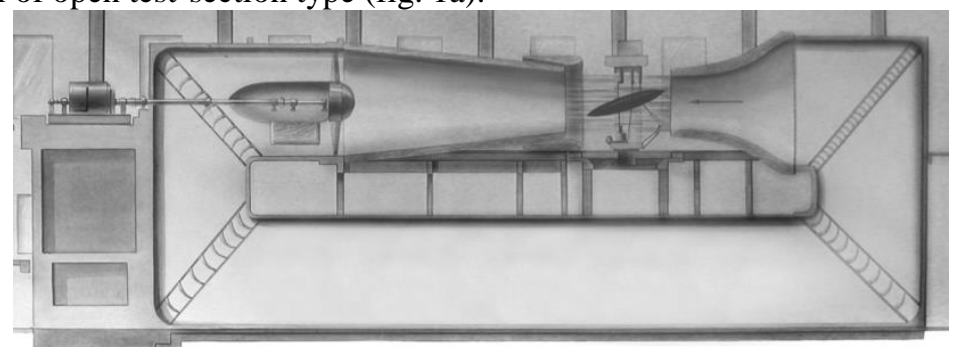

a

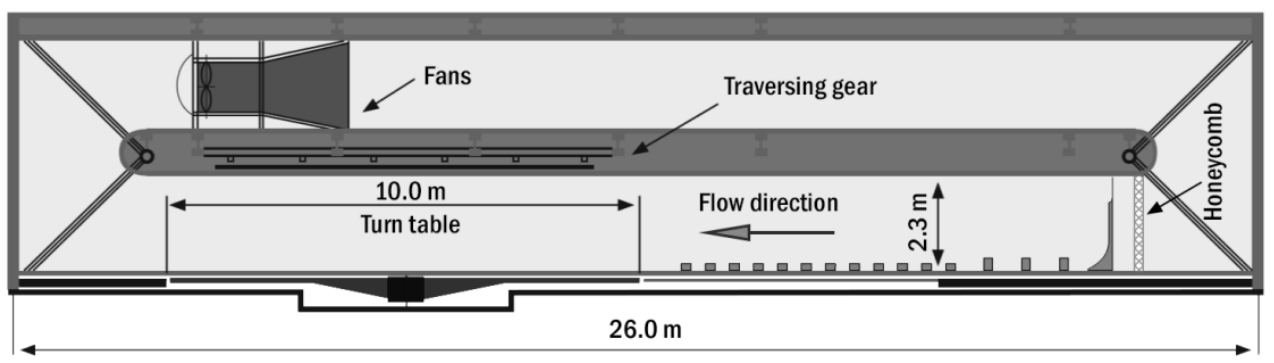

b

Fig. 1. Scheme of the wind tunnels of Krylov State Research Centre $\mathrm{a}$ - wind tunnel with open test section; $\mathrm{b}$ - LWT

Then, a scaled elastic similar model of the entire bridge is made to examine its overall aerodynamic stability. This one is tested in a special wind tunnel with a wide test section, sometimes incorporating models of relevant background structures and landscape features [14-16]. A set of requirements have to be met regarding the scale and manufacturing tolerances of models as well as the wind tunnel facilities simulating air flow to enable correct physical modelling of long-span bridge aerodynamics. The wind tunnel test section is to be long enough to simulate the ground boundary layer, and wide enough to accommodate large-scale bridge models with surrounding terrain features.

All these requirements for aerodynamic experiments on long-span bridges are satisfied in the Landscape Wind Tunnel (LWT) of Krylov State Research Centre (fig. 1b).

LWT Main Data:

- closed-circuit wind tunnel with closed test section;

- test section shape - rectangular $11 \times 2,3 \mathrm{~m}$;

- test section length - $18 \mathrm{~m}$;

- incident flow speed- up to $14 \mathrm{~m} / \mathrm{s}$;

- flow speed adjustment step- $0.1 \mathrm{~m} / \mathrm{s}$.

\section{Results and Discussion}

As a case study let us consider the aero-elastic stability of a $420 \mathrm{~m}$ cable stayed bridge sized $80 \times 260 \times 80$ (fig. 2a). This bridge has a reinforced concrete span structure of $16,7 \mathrm{~m}$ width, cable-stayed bridge of $72.3 \mathrm{~m}$ height (fig. $2 \mathrm{~b}$ ). 


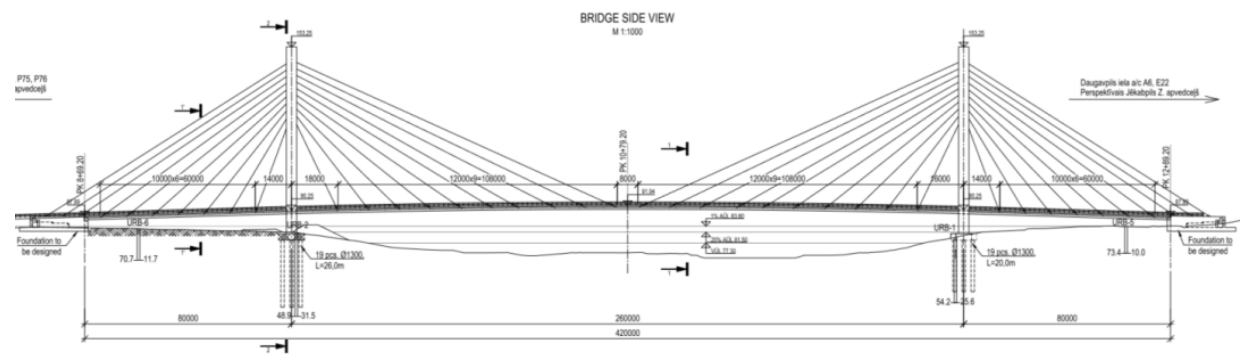

a

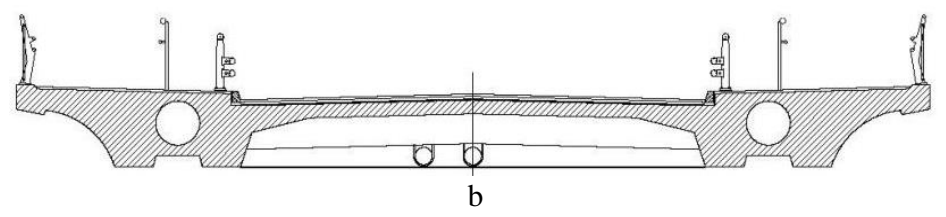

Fig. 2. Scheme of typical cable stayed bridge $\mathrm{a}$ - general view; $\mathrm{b}$ - croos-section

These studies were done in two steps. First, a dynamically similar section model of cable bridge was made (fig. 3) to 1:35 scale. This model enables prompt examination of the span structure stability in various service situations considering:

- initial design of bridge structure;

- motor traffic;

- snow on guardrails;

- acoustic shields.

The above-said tests were performed in laminar flow conditions at a number of different flow directions in vertical plane. When bridge span oscillations were detected, the same was tested in turbulent flow conditions.

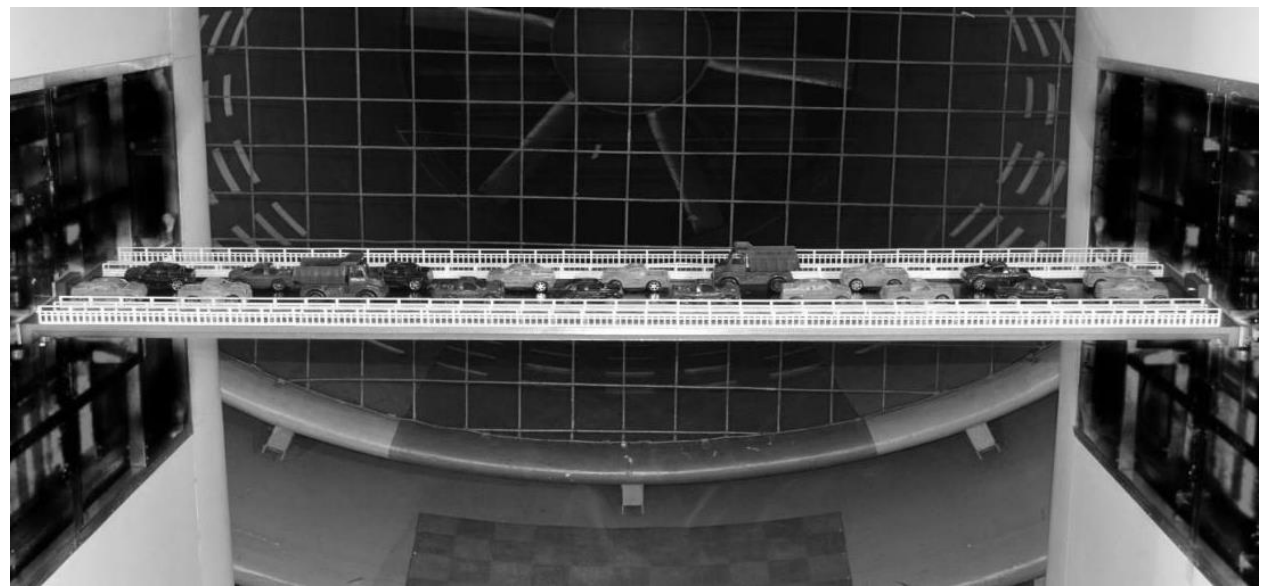

Fig. 3. Section model of cable bridge mounted on dynamic rig in wind-tunnel test section. Bridge model with motor traffic

Model tests with snow cover showed vertical oscillations due to aero-elastic instability excited by vortices. The critical velocity giving rise to maximum RMS amplitudes was 7 to $8 \mathrm{~m} / \mathrm{s}$ (see fig. $4 \mathrm{a}$, all data scaled up to full size). The model configuration with snow cover was also subject to torsional oscillations due to torsional flutter instability. This type of oscillations started from $20 \mathrm{~m} / \mathrm{s}$ at wind directions $\alpha=5^{\circ}$, and were growing indefinitely with the wind velocity (fig. $4 \mathrm{~b}$ ), where the angle $\alpha$ counts rotation relative to the 
longitudinal axis of the bridge. When the tests were repeated in turbulent flow, the maximum amplitudes diminished.
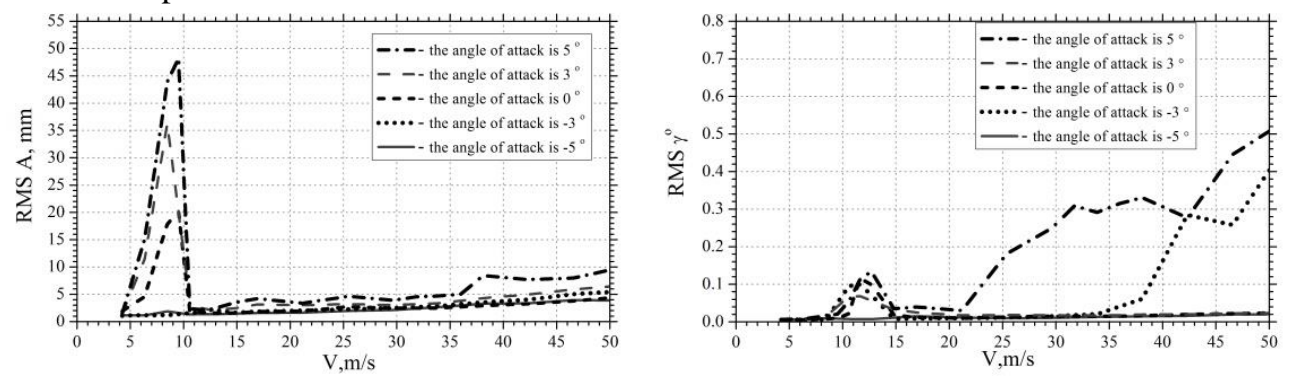

Fig. 4. RMS amplitudes

$\mathrm{a}-$ vertical bridge oscillations versus wind velocity. Bridge with snow cover; $\mathrm{b}$ - torsional bridge oscillations versus wind velocity. Bridge with snow cover

The second step was to verify the aero-elastic stability of the whole bridge structure including pylons and stay cables, rather than the span section alone. An elastically similar model of the complete bridge was made to 1:75 scale. Prior to manufacture, the full-scale structure characteristics were scaled down to model size based on the similarity criteria. The bridge model is made up of two main parts: a metal rod of variable section for modeling the stiffness properties and a shell for modeling geometry as well as mass and inertial characteristics.

Special attention should be paid to proper modelling of the stay cable system. Stay cables of a real bridge are sheathed steel ropes bracing the pylon and the span structure. The stay cables have certain stiffness related to material properties and geometry of suspension ropes. Also, stay cables have their own aerodynamic drag, which under wind action would influence the overall bridge behaviour. Direct extrapolation of geometric dimensions from full size to model scale would not ensure similarity. Therefore, stay cables are modelled as built-up structures incorporating stiffness and drag simulation elements. Stiffness of fullscale cables was modelled using scale factors. Then, an auxiliary spring was selected with proper consideration of the cable stiffness. Using this procedure each bridge cable was modelled with an individual spring whose stiffness was within $3 \%$ of the target value. Aerodynamic drag of stay cables was modelled with additional elements fixed directly on model suspension ropes (fig.5a).

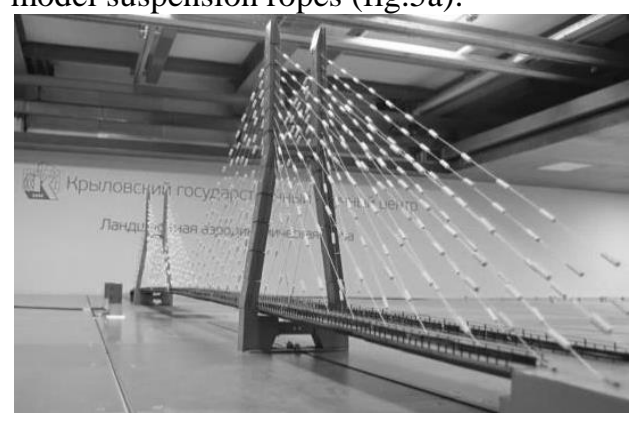

a

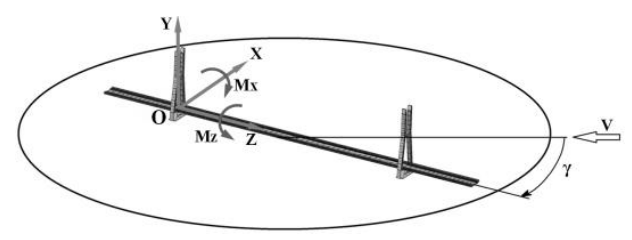

b

Fig. 5. Elastic model of entire bridge

$\mathrm{a}$ - photo in the LWT; $\mathrm{b}$ - coordinate system connected with bridge model

Tests were conducted at each heading with stepwise velocity increase. Coordinate system connected with model is shown at the fig. $5 \mathrm{~b}$. The tested full-scale wind velocities covered a range of 0 to $52 \mathrm{~m} / \mathrm{s}$ with an increment of $1 \mathrm{~m} / \mathrm{s}$ at the bridge span height. This range of wind velocities was chosen based on weather data records available for the bridge location and specified as design inputs. Table 4 contains the initial data inputs on wind velocities in the area under consideration $[5,6]$. 
Table 1. Regulatory and design wind velocity data for the bridge construction area (initial data)

\begin{tabular}{|c|c|}
\hline Velocity [m/s] & Value \\
\hline Regulatory wind velocity & 25.7 \\
\hline Design wind velocity & 31.4 \\
\hline Test velocity for aerodynamic stability & 52.0 \\
\hline
\end{tabular}

Displacements of the bridge span and pylon model were measured with eight optical sensors. Four sensors were used to measure vertical oscillations at mid- and quarter-length of the central span section. Measurements were taken at two symmetrical points with respect to the bridge longitudinal axis. These measurements enabled us to estimate displacements and amplitudes of vertical and torsional oscillations at the bridge's central span model.

Two sensors measured horizontal displacements at mid- and quarter-length of the central span section. These measurements provided inputs to estimate displacements and amplitudes of horizontal oscillations at the central span model. Two more sensors ware used to measure horizontal displacements and accelerations at the top of pylon struts in two mutually perpendicular planes. The same sensors measured accelerations at the top of pylon struts.

The test data on the aerodynamic stability of elastically similar model of cable bridge are presented as displacements (with reference to the body-fixed axes) in function of fullscale wind velocities and as root-mean-square amplitudes of oscillations (with reference to the same axes) in function of full-scale wind velocities (fig. 6).

The relationships of $\Delta \mathrm{X}_{\mathrm{H}}, \Delta \mathrm{Y}_{\mathrm{H}}$ and $\Delta \mathrm{Z}_{\mathrm{H}}$ displacements with reference to body axes versus full-scale wind velocity are presented as standard deviation of mean displacements from the initial position at velocity increment.

Root-mean-square oscillation amplitudes RMS $X_{H}$, RMS $Y_{H}$ and RMS $Z_{H}$ versus fullscale wind velocity are expressed as square root of measured value variance from the following formula

$$
R M S X_{H}=\sqrt{D\left(X_{H}\right)},
$$

where $R M S X_{H}$ - root-mean-square value of oscillation amplitude at the reference point in $\mathrm{m} ; D\left(X_{H}\right)$ - variance of oscillations at the reference point.

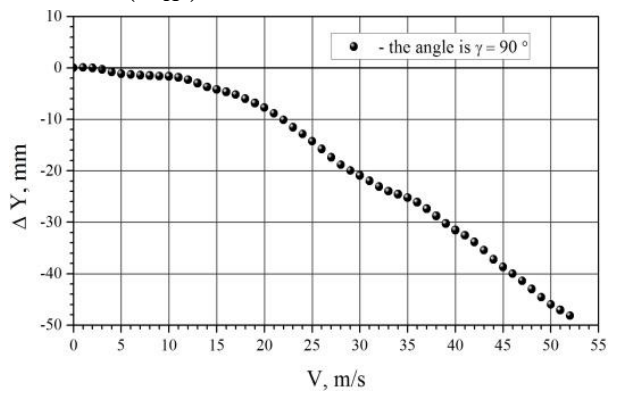

a

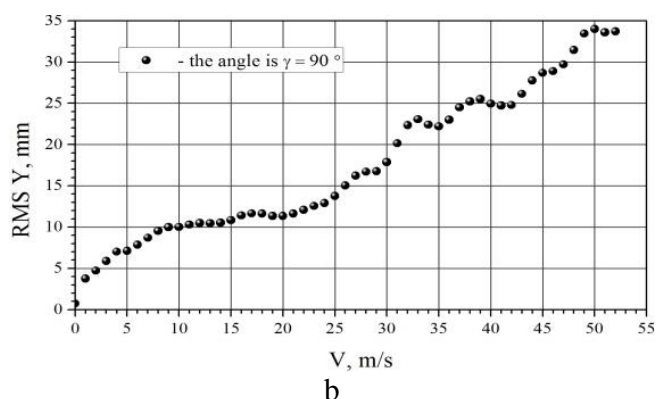

b

Fig. 6. Characteristics of vertical oscillations versus incident flow velocity $\mathrm{a}-$ at the mid-length point of central span; $\mathrm{b}$ - RMS amplitude at bridge span mid-length

Experiments with the elastically similar model of cable stayed bridge have not identified any dangerous oscillations of the bridge, therefore it is concluded that the structure is aerodynamically stable. 


\section{Conclusions}

Bridge structures with spans over 100 meters are quite flexible and could be unstable under wind loads [17-23]. At present physical model experiments in wind tunnels remain the most reliable and convenient method for detection of bridge oscillation risks early in the design process. This paper describes a case study on aerodynamic stability of a bridge span structure using a dynamically similar model of the bridge span section as well as an elastically similar model of the whole bridge structure. Tests of the span section model enabled prompt and simple examination of all possible bridge service situations (initial configuration, modelling of snow cover, motor traffic, etc.) and identify oscillations. Tests of the elastically similar model of the whole bridge structure led to the conclusion of the overall aerodynamic stability of the bridge.

\section{References}

1. Y. Fujino, Vibration, control and monitoring of long-span bridges-recent research, developments and practice in Japan. J. of Const. Steel Research, 58, 71-97 (2002)

2. F. B. Farquharson, S. F. Smith, Aerodynamic stability of suspension bridges, for example bridge across the strait Tacoma. Proceedings of the University of Washington, 116, (1954)

3. J. B. Fradsen, Simultaneous pressures and accelerations measured full-scale on the Great Belt East suspension bridge. J. Wind Eng. Ind. Aerodym, 89, 95-129 (2001)

4. M. Yu et al., Field measurement and wind tunnel test of buffeting response of longspan bridge under skew wind. J. of Exp. Fluid Mech., 27, 51-55 (2013)

5. Cun-ming Ma et al., KSCE J. Civ. Eng., 1-10 (2017)

6. Z. Taylor et al., J. Struct. Eng., 143, 11 (2017)

7. J.S. Lee, KSCE J. Civ. Eng., 4, 191-199 (2000)

8. A. Hirai, J. of the Japanese soc. of civil engineers, 28, (1942)

9. M.-H. Huang et al., J. App. Science and Eng., 15, 401-413 (2012)

10. Z. T. Zhang et al., J. Fluid Str., 40, 269-283 (2013)

11. C. Costa et. al., J. Wind Eng. Ind. Aerodym., 95, 991-1006 (2007)

12. Q. Zhu, Y. L. Xu, J. Wind Eng. Ind. Aerodym., 131, 31-45 (2014)

13. K. C. S. Kwok et al., J. Wind Eng. Ind. Aerodym., 110, 50-61 (2012)

14. J. Counihan, Atmospheric Environment, 3, 197-214 (1967)

15. J. Armitt, J. Counihan, Atmospheric Environment, 2, 49-71 (1968)

16. S. Solovev, Aerodynamic stability of large-span bridges. Transport of the Russian Federation, 5, (2016)

17. Eurocode 1: Actions on structures - 1-4: General actions - Wind actions

18. SP 20.13330.2016 - Loads and actions

19. B. Kovačič, A. Marendić, R. Kamnik, M. Volkov, V. Roy, MATEC Web Conf, 73 (2016)

20. S. Ognjenovic, T. Sazonova, R. Ristov, I. Nedevska, A. Kibkalo, MATEC Web Conf., 73, (2016)

21. N. Bobylev, Tunn. Undergr., 55, 40-51 (2016).

22. R.V. Vvedenskij, S.G. Gendler, T.S. Titova, Mag. Civ. Eng., 79(3), (2018)

23. V.A. Schegolev, O.V. Novoseltsev, Teoriya i Praktika Fizicheskoy Kultury (2015) 